\title{
Fringe-Order Determination Method in White-Light Phase-Shifting Interferometry for the Compensation of the Phase Delay and the Suppression of Excessive Phase Unwrapping
}

\author{
SeongRyong Kim*, JungHwan Kim, and HeuiJae Pahk \\ School of Mechanical and Aerospace Engineering, Seoul National University, Seoul 151-747, Korea
}

(Received July 16, 2013 : revised September 23, 2013 : accepted September 24, 2013)

\begin{abstract}
White-light phase-shifting interferometry (WLPSI) is widely recognized as a standard method to measure shapes with high resolution over a long distance. In practical applications, WLPSI, however, is associated with some degree of ambiguity of its phase, which occurs due to a phase delay, which is the offset between the phase of the fringes and the fringe envelope peak position. In this paper, a new algorithm is proposed for the determination of a fringe order suitable for samples in which the phase delay mainly occurs due to noise, diffraction and a steep angle. The concepts of the decouple factor and the connectivity are introduced and a method for calculating the decouple factor and the connectivity is developed. With the phase-unwrapping procedure which considers these values, it is demonstrated that our algorithm determines the correct fringe order. To verify the performance of the algorithm, a simulation was performed with the virtual step height under noise. Some specimens such as step height standard and a column spacer with a steep angle are also measured with a Mirau interference microscope, after which the algorithm is shown to be effective and robust.
\end{abstract}

Keywords: White light interferometry, Fringe order determination, Phase unwrapping, Decouple factor, Connectivity

OCIS codes : (120.0120) Instrumentation, measurement, and metrology; (120.3180) Interferometry

\section{INTRODUCTION}

White-light phase-shifting interferometry (WLPSI) proposed by [2] and [3,4] has high resolution over long distances. WLPSI, however, cannot overcome the limitation of phaseambiguity entirely, because it can also detect the fringe order incorrectly due to the problem of phase ambiguity. In order to address this issue, a specific strategy has been required to determine the correct fringe order in relation to WLPSI.

Previous research related to determining of fringe order was done by [3, 4], [5, 6] and [7]. This work, however, yielded solutions only for limited samples. [8] suggested a method to determine the fringe order by phase unwrapping, though it is not sufficient in practical applications given undesirable conditions such as noise, diffraction and steep angles.

In this paper, a new algorithm that corrects errors in fringe-order determinations is proposed. Prior to the phase unwrapping process, parameters such as the decouple factor and the connectivity are introduced and calculated. The decouple factor can compensate for the effect of a phase delay. As the connectivity contains information on the edges at which phase unwrapping is prohibited, it is possible to suppress excessive phase unwrapping.

To verify the performance of the algorithm, a simulation was performed with the virtual step height under noise. Some specimens such as step height standard and a column spacer with a steep angle are also measured with a Mirau interference microscope, after which the algorithm is shown to be effective and robust.

\section{METHODS}

\subsection{Introduction of the Decouple Factor}

The WLPSI equation using both the phase of white-light

\footnotetext{
*Corresponding author: srkim1303@gmail.com

Color versions of one or more of the figures in this paper are available online.
} 
scanning interferometry (WSI) and the phase of phase shifting interferometry (PSI) is

$$
\phi_{w l p s i}(i)=\phi_{w s i}(i)+\phi_{p s i}(i), \text { for the i-th pixel }
$$

of the image.

where $\phi_{w l p s i}$ is the phase from WLPSI, $\phi_{w s i}$ is the phase of the fringe envelope peak position, and $\phi_{p s i}$ is the phase of the fringe relative to the $\phi_{w s i}$ position. When the phase delay, which is the offset between the phase of the fringes and the fringe envelope peak position is greater than $\pi, \phi_{p s i}$ cannot be calculated correctly due to the ambiguity of the phase. We can then only calculate $\tilde{\phi}_{p s i}$ between $-\pi$ and $\pi$ as shown in Fig. 1. This means that $\phi_{p s i}$ and $\tilde{\phi}_{p s i}$ can be related as follows;

$$
\phi_{p s i}(i)=\tilde{\phi}_{p s i}(i)+2 n \pi, \quad n=\text { integer }
$$

When we assume the fringe order $n$ to be zero, the equation for WLPSI, Eq. (1), can be written as:

$$
\tilde{\phi}_{w l p s i}(i)=\phi_{w s i}(i)+\tilde{\phi}_{p s i}(i)
$$

where $\tilde{\phi}_{w l p s i}$ is the phase from WLPSI when the fringe order $n$ is assumed to be zero.

From Eq. (3), a reasonable proposition can be made such that the fringe order $\tilde{\phi}_{w l p s i}$ can be assigned as the same fringe order of $\tilde{\phi}_{p s i}$, when the both sides of Eq. (3) are phase-unwrapped. We can express this condition as

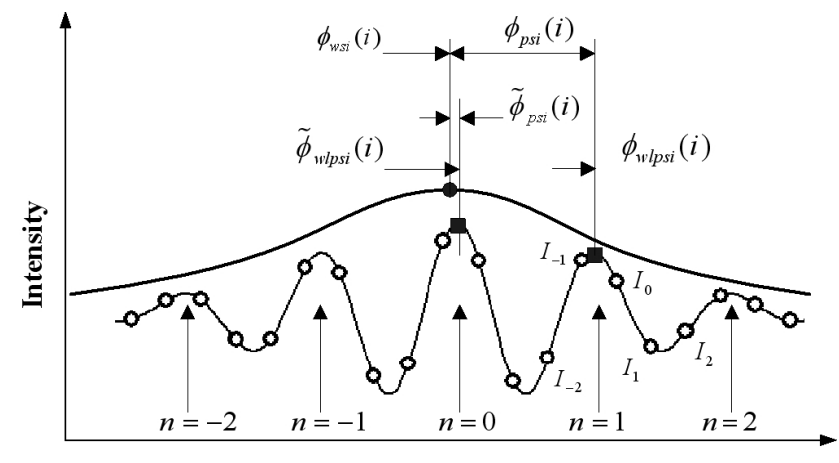

Phase

FIG. 1. Phase definition of the interference signal for a low-coherence source. The upper line is the envelope of interference signal. The black circle point is the peak of the envelope which is WSI the phase. The lower line is the interference signal. The blank circle points denote the captured signal when we use a 4 bucket or 5 bucket algorithm for calculating the phase. The left rectangle point is the phase, which is calculated with the incorrect fringe-order determination. The right rectangle point indicates the correct phase. follows;

For neighboring points,

$$
\begin{gathered}
(2 m-1) \pi<\left(\tilde{\phi}_{w l p s i}(i+1)-\tilde{\phi}_{w l p s i}(i)\right) \leq(2 m+1) \pi \\
(2 m-1) \pi<\left(\tilde{\phi}_{p s i}(i+1)-\tilde{\phi}_{p s i}(i)\right) \leq(2 m+1) \pi(4) \\
m=\text { integer }
\end{gathered}
$$

where $m$ is the integer of the fringe-order difference between two neighboring points.

Equation (4), however, cannot be met at all points due to practical measurement conditions such as noise, diffraction and $\mathrm{s}$ steep angle. Therefore, $\phi_{d}$, a decouple factor, can be introduced to address this issue:

$$
\begin{aligned}
\phi_{w s i, d}(i) & =\phi_{w s i}(i)+\phi_{d}(i) \\
\tilde{\phi}_{p s i, d}(i) & =\widetilde{\phi}_{p s i}(i)-\phi_{d}(i)
\end{aligned}
$$

where $\phi_{w s i}$ and $\tilde{\phi}_{p s i}$ are compensated as $\phi_{w s i, d}$ and $\tilde{\phi}_{p s i, d}$, respectively by the decouple factor $\phi_{d}$. Then Eq. (3) can be rewritten using Eq. (5).

$$
\tilde{\phi}_{w l p s i}(i)=\phi_{w s i, d}(i)+\tilde{\phi}_{p s i, d}(i)
$$

If we can calculate $\phi_{d}$ which makes $\phi_{w s i, d}$ and $\tilde{\phi}_{p s i, d}$ satisfy Eq. (4) at all points, the unwrapping method can become more robust to determine the fringe order.

\subsection{Calculation of the Decouple Factor}

In this section, we propose a method to calculate the decouple factor in practical applications. From our practical application, $\phi_{w s i, d}$ can be considered such that the difference in $\phi_{w s i, d}$ between the neighboring points falls within the range of $-\pi$ and $\pi$. In addition, the fringe-order difference $m$ can be limited between -2 and 2 such that the phase delay due to undesirable conditions such as noise and diffraction are limited between $-5 \pi$ and $5 \pi$.

Then Eq. (4) can be rewritten considering Eq. (5) and Eq. (6).

$$
\begin{gathered}
(2 m-1) \pi<\left(\tilde{\phi}_{w l p s i}(i+1)-\tilde{\phi}_{w l p s i}(i)\right) \leq(2 m+1) \pi \\
\left\{\begin{array}{c}
-\pi<\left(\phi_{w s i, d}(i+1)-\phi_{w s i, d}(i)\right) \leq \pi \\
(2 m-1) \pi<\left(\tilde{\phi}_{p s i, d}(i+1)-\tilde{\phi}_{p s i, d}(i)\right) \leq(2 m+1) \pi
\end{array}\right\} \\
m=-2,-1,0,1,2
\end{gathered}
$$

After Eq. (5) is applied to Eq. (7),

$$
\left\{\begin{array}{c}
-\pi<\left(\phi_{w s i}(i+1)+\phi_{d}(i+1)-\phi_{w s i, d}(i)-\phi_{d}(i)\right) \leq \pi \\
(2 m-1) \pi<\left(\tilde{\phi}_{p s i}(i+1)-\phi_{d}(i+1)-\tilde{\phi}_{p s i}(i)+\phi_{d}(i)\right) \leq(2 m+1) \pi
\end{array}\right\}
$$


Let $\Delta \phi_{d}(i)$ be the difference in the decouple factor between the neighboring points,

$$
\Delta \phi_{d}(i)=\phi_{d}(i+1)-\phi_{d}(i)
$$

Apply Eq. (9) into Eq. (8),

$$
\left\{\begin{array}{c}
\left.-\pi<\left(\left(\phi_{w s i}(i+1)-\phi_{w s i, d}(i)\right)+\Delta \phi_{d}(i)\right)\right) \leq \pi \\
(2 m-1) \pi<\left(\left(\tilde{\phi}_{p s i}(i+1)-\tilde{\phi}_{p s i}(i)\right)-\Delta \phi_{d}(i)\right) \leq(2 m+1) \pi
\end{array}\right\}(10)
$$

From Eq. (10), $\Delta \phi_{d}(i)$ can be ranged as follows;

$$
\begin{gathered}
\left\{\begin{array}{c}
-\pi-A(i)<\Delta \phi_{d}(i) \leq \pi-A(i) \\
-(2 m+1) \pi+B(i) \leq \Delta \phi_{d}(i)<-(2 m-1) \pi+B(i)
\end{array}\right\} \\
A(i)=\phi_{w s i}(i+1)-\phi_{w s i}(i) \\
B(i)=\tilde{\phi}_{p s i}(i+1)-\tilde{\phi}_{p s i}(i)
\end{gathered}
$$

For a valid $\Delta \phi_{d}(i)$ in practical application, the range of $\Delta \phi_{d}(i)$ can be obtained between the maximum of the two left-hand limits and the minimum of the two right-hand limits of Eq. (11),

$$
\max \left(\begin{array}{c}
-\pi-A(i) \\
-(2 m+1) \pi+B(i)
\end{array}\right)<\Delta \phi_{d}(i)<\min \left(\begin{array}{c}
\pi-A(i) \\
-(2 m-1) \pi+B(i)
\end{array}\right)
$$

In order to calculate the decouple factor $\phi_{d}(i)$, an iteration procedure is used. The initial $\overline{\Delta \phi_{d}}(i)$ can be assigned as the median value of the limits of Eq. (12). That is,

$$
\overline{\Delta \phi_{d}}(i)=\frac{1}{2}\left(\begin{array}{c}
\max (-\pi-A(i),-(2 m+1) \pi+B(i)) \\
+\min (\pi-A(i),-(2 m-1) \pi+B(i))
\end{array}\right)(13)
$$

$\overline{\Delta \phi_{d}}(i)$ will be used instead of $\Delta \phi_{d}(i)$ in the subsequent calculations.

For an easy explanation, we consider the pixel and edge figure shown in Fig. 2. The pixel figure indicates the value

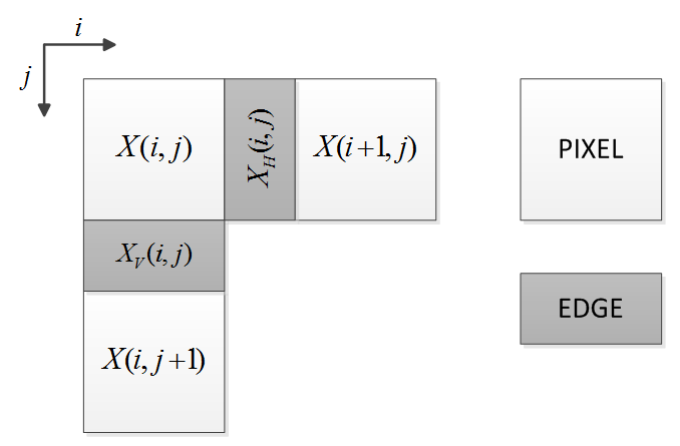

FIG. 2. Definition of a pixel and an edge. We define the edge to explain the connectivity, which is defined between neighboring pixels. defined at the pixel, in this case $\tilde{\phi}_{w l p s i}, \tilde{\phi}_{p s i}$, and $\phi_{d}$. The edge figure indicates the difference value between the pixel values, in this case $\Delta \phi_{d}, A$, and $B$ in Eq. (9) and (11).

We extend the previous equations from a one dimensional case to a two dimensional case as shown in Fig. 3. In a two dimensional case, one point has four edges on the upper, lower, left, and right sides. The difference values, $A(i), B(i)$, Eq. (13) and $\Delta \phi_{d}(i)$ can be rewritten as follows;

$$
\begin{aligned}
& \mathrm{A}_{H}(i, j)=\phi_{w s i}(i+1, j)-\phi_{w s i}(i, j) \\
& \mathrm{A}_{V}(i, j)=\phi_{w s i}(i, j+1)-\phi_{w s i}(i, j) \\
& \mathrm{B}_{H}(i, j)=\tilde{\phi}_{p s i}(i+1, j)-\tilde{\phi}_{p s i}(i, j) \\
& \mathrm{B}_{V}(i, j)=\tilde{\phi}_{p s i}(i, j+1)-\tilde{\phi}_{p s i}(i, j) \\
& (2 m-1) \pi<\left(\tilde{\phi}_{w l p s i}(i+1, j)-\tilde{\phi}_{w l p s i}(i, j)\right) \leq(2 m+1) \pi \\
& \left\{-\pi-A_{H}(i, j)<\Delta_{H} \phi_{d}(i, j) \leq \pi-A_{H}(i, j)\right. \\
& \left\{-(2 m+1) \pi+B_{H}(i, j) \leq \Delta_{H} \phi_{d}(i, j)<-(2 m-1) \pi+B_{H}(i, j)\right\} \\
& m=-2,-1,0,1,2 \\
& (2 m-1) \pi<\left(\tilde{\phi}_{w l p s i}(i, j+1)-\tilde{\phi}_{w l p s i}(i, j)\right) \leq(2 m+1) \pi \\
& \left\{\begin{array}{c}
-\pi-A_{V}(i, j)<\Delta_{V} \phi_{d}(i, j) \leq \pi-A_{V}(i, j) \\
-(2 m+1) \pi+B_{V}(i, j) \leq \Delta_{V} \phi_{d}(i, j)<-(2 m-1) \pi+B_{V}(i, j)
\end{array}\right\} \\
& m=-2,-1,0,1,2 \\
& \overline{\Delta_{H} \phi_{d}}(i, j)=\frac{1}{2}\left(\begin{array}{l}
\max \left(-\pi-A_{H}(i, j),-(2 m+1) \pi+B_{H}(i, j)\right) \\
+\min \left(\pi-A_{H}(i, j),-(2 m-1) \pi+B_{H}(i, j)\right)
\end{array}\right) \\
& \overline{\bar{S}_{V} \phi_{d}}(i, j)=\frac{1}{2}\left(\begin{array}{c}
\max \left(-\pi-A_{V}(i, j),-(2 m+1) \pi+B_{V}(i, j)\right) \\
+\min \left(\pi-A_{V}(i, j),-(2 m-1) \pi+B_{V}(i, j)\right)
\end{array}\right)
\end{aligned}
$$

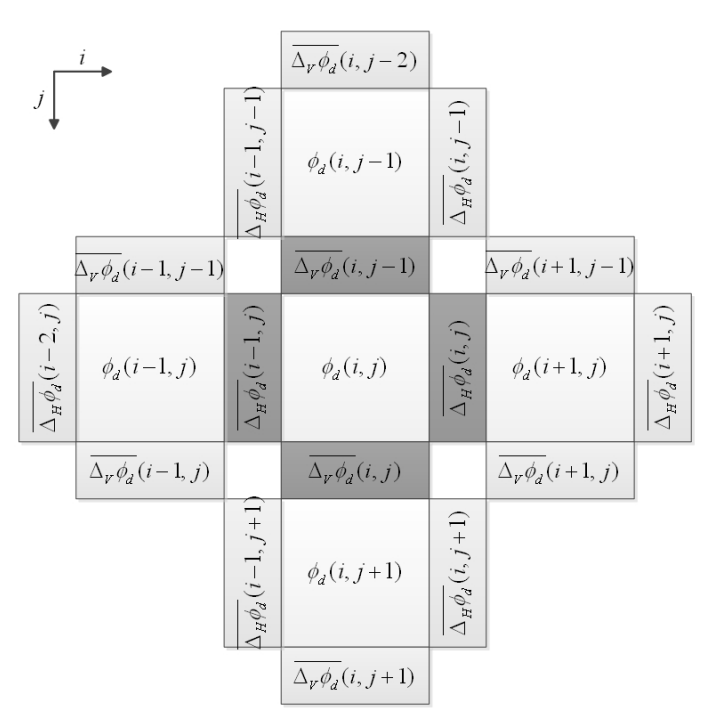

FIG. 3. Decouple factor in a two dimensional case. This figure shows how the definition of the decouple factor changes in a two dimensional case. At the pixel, the decouple factor is defined. At the edge, the difference of the decouple factor is defined. 


$$
\begin{aligned}
& \Delta_{H} \phi_{d}(i, j)=\overline{\Delta_{H} \phi_{d}}(i, j)=\phi_{d}(i+1, j)-\phi_{d}(i, j) \\
& \Delta_{V} \phi_{d}(i, j)=\overline{\Delta_{V} \phi_{d}}(i, j)=\phi_{d}(i, j+1)-\phi_{d}(i, j)
\end{aligned}
$$

We already know the values of $\phi_{w s i}$ and $\tilde{\phi}_{p s i}$ at all pixels. Using Eq. (14), (15), and (18), we can calculate the values of $\overline{\Delta_{H} \phi_{d}}(i, j)$ at all edges.

Next, we explain how to calculate the decouple factor $\phi_{d}$ from the difference of the decouple factor $\overline{\Delta \phi_{d}}(i)$. In Fig. 3, the values of $\Delta \phi_{d}$ in the four gray edges are written as follows;

$$
\left\{\begin{array}{c}
\Delta_{H} \phi_{d}(i-1, j)=\phi_{d}(i, j)-\phi_{d}(i-1, j) \\
\Delta_{H} \phi_{d}(i, j)=\phi_{d}(i+1, j)-\phi_{d}(i, j) \\
\Delta_{V} \phi_{d}(i, j-1)=\phi_{d}(i, j)-\phi_{d}(i, j-1) \\
\Delta_{V} \phi_{d}(i, j)=\phi_{d}(i, j+1)-\phi_{d}(i, j)
\end{array}\right.
$$

Each equation has one $\phi_{d}(i, j)$. Let $\phi_{d}(i, j)$ denote the average of four $\phi_{d}(i, j)$ in Eq. (20). This leads to Eq. (21).

$$
\begin{aligned}
\phi_{d}(i, j)= & \frac{1}{4}\left(\begin{array}{c}
\Delta_{H} \phi_{d}(i-1, j)-\Delta_{H} \phi_{d}(i, j) \\
+\Delta_{V} \phi_{d}(i, j-1)-\Delta_{V} \phi_{d}(i, j)
\end{array}\right) \\
& +\frac{1}{4}\left(\begin{array}{c}
\phi_{d}(i-1, j)+\phi_{d}(i+1, j) \\
+\phi_{d}(i, j-1)+\phi_{d}(i, j+1)
\end{array}\right)
\end{aligned}
$$

After this strategy is applied once more to the second term in Eq. (21), Eq. (21) is rewritten as follows;

$$
\begin{aligned}
\phi_{d}(i, j)= & \frac{1}{4}\left(\begin{array}{c}
\Delta_{H} \phi_{d}(i-1, j)-\Delta_{H} \phi_{d}(i, j) \\
+\Delta_{V} \phi_{d}(i, j-1)-\Delta_{V} \phi_{d}(i, j)
\end{array}\right) \\
& +\frac{1}{16}\left(\begin{array}{c}
\Delta_{H} \phi_{d}(i-2, j)-\Delta_{H} \phi_{d}(i-1, j) \\
+\Delta_{V} \phi_{d}(i-1, j-1)-\Delta_{V} \phi_{d}(i-1, j) \\
+\Delta_{H} \phi_{d}(i-1, j-1)-\Delta_{H} \phi_{d}(i, j-1) \\
+\Delta_{V} \phi_{d}(i, j-2)-\Delta_{V} \phi_{d}(i, j-1) \\
+\Delta_{H} \phi_{d}(i, j)-\Delta_{H} \phi_{d}(i+1, j) \\
+\Delta_{V} \phi_{d}(i+1, j-1)-\Delta_{V} \phi_{d}(i+1, j) \\
+\Delta_{H} \phi_{d}(i-1, j+1)-\Delta_{H} \phi_{d}(i, j+1) \\
+\Delta_{V} \phi_{d}(i, j)-\Delta_{V} \phi_{d}(i, j+1)
\end{array}\right) \\
& +\frac{1}{64}(\ldots)
\end{aligned}
$$

The third term in Eq. (22) has a constant $\frac{1}{64}$. This effect is almost $1 \%$ of the total effect. This term can be ignored.

\subsection{Introduction of the Concept of Connectivity}

We introduce the concept of connectivity in order to consider the disconnected edges, serving two purposes. First, the connectivity can change the decouple factor $\phi_{d}(i, j)$ to satisfy Eq. (12). Second, this prohibits the unwrapping of the phase at the disconnected edges.

The connectivity can be given at all edges as follows;

$$
\begin{gathered}
C_{H}(i, j) \begin{cases}1 & (i+1, j) \text { and }(i, j) \text { are connected } \\
0 & \text { otherwise }\end{cases} \\
C_{V}(i, j) \begin{cases}1 & (i, j+1) \text { and }(i, j) \text { are connected } \\
0 & \text { otherwise }\end{cases}
\end{gathered}
$$

The connectivity initially is assigned as one at all edges, and then changed to a zero under the next three conditions

1. At the boundary of the measurement area: that is, $i=0, i=M_{x} ; j=0, j=M_{y}$.

2. At the edges of an point where the light intensity signal cannot be obtained.

3. If the difference in $\tilde{\phi}_{w l p s i}$ between the neighboring points exceed over $5 \pi$, as mentioned in relation to Eq. (7).

where $M_{x}$ is the horizontal image size and $M_{y}$ is the vertical image size. When we consider connectivity, the equation for the decouple factor, Eq. (22), is changed, as follows;

$$
\begin{gathered}
\phi_{d}(i, j)=\frac{1}{4}\left(\begin{array}{c}
C_{H}(i-1, j) \Delta_{H} \phi_{d}(i-1, j)-C_{H}(i, j) \Delta_{H} \phi_{d}(i, j) \\
+C_{V}(i, j-1) \Delta_{V} \phi_{d}(i, j-1)-C_{V}(i, j) \Delta_{V} \phi_{d}(i, j)
\end{array}\right) \\
+\frac{1}{16}\left(\begin{array}{c}
C_{H}(i-2, j) C_{H}(i-1, j) \Delta_{H} \phi_{d}(i-2, j) \\
-C_{H}(i-1, j) \Delta_{H} \phi_{d}(i-1, j) \\
+C_{V}(i-1, j-1) C_{H}(i-1, j) \Delta_{V} \phi_{d}(i-1, j-1) \\
-C_{V}(i-1, j) C_{H}(i-1, j) \Delta_{V} \phi_{d}(i-1, j) \\
+C_{H}(i-1, j-1) C_{V}(i, j-1) \Delta_{H} \phi_{d}(i-1, j-1) \\
-C_{H}(i, j-1) C_{V}(i, j-1) \Delta_{H} \phi_{d}(i, j-1) \\
+C_{V}(i, j-2) C_{V}(i, j-1) \Delta_{V} \phi_{d}(i, j-2) \\
-C_{V}(i, j-1) \Delta_{V} \phi_{d}(i, j-1) \\
+C_{H}(i, j) \Delta_{H} \phi_{d}(i, j) \\
-C_{H}(i+1, j) C_{H}(i, j) \Delta_{H} \phi_{d}(i+1, j) \\
+C_{V}(i+1, j-1) C_{H}(i, j) \Delta_{V} \phi_{d}(i+1, j-1) \\
-C_{V}(i+1, j) C_{H}(i, j) \Delta_{V} \phi_{d}(i+1, j) \\
+C_{H}(i-1, j+1) C_{V}(i, j) \Delta_{H} \phi_{d}(i-1, j+1) \\
-C_{H}(i, j+1) C_{V}(i, j) \Delta_{H} \phi_{d}(i, j+1) \\
+C_{V}(i, j) \Delta_{V} \phi_{d}(i, j) \\
-C_{V}(i, j+1) C_{V}(i, j) \Delta_{V} \phi_{d}(i, j+1)
\end{array}\right)
\end{gathered}
$$

\subsection{Iterative Comection of the Decouple Factor and the Connectivity}

We check and correct the decouple factor and the connectivity using the steps in Fig. 4 and Fig. 5, where Fig. 4 shows a flow chart for checking the decouple factor and changing the connectivity at each pixel and Fig. 5 shows when using this flow chart. First, $\phi_{d}(i, j)$ is calculated by Eq. (24) and the connectivity at the first pixel in Fig. 5. If the range conditions, Eq. (16) and (17), of the difference in $\phi_{d}(i, j)$ are not satisfied or if the connectivity of the edges is not disconnected, the connectivity at the edge where the difference in $\tilde{\phi}_{w l p s i}$ is greatest among the four edges is changed to "disconnected". Then, $\phi_{d}(i, j)$ is re-calculated with the updated connectivity. The reason to check only the range condition, the Eq. (16) and (17), at 
the left edge and the upper edge is that checking at the right edge and the bottom edge will be processed at the next pixels. For example, when the decouple factor and the connectivity at pixel 5 was checked as shown in Fig. 5, we don't check at the right edge and the bottom edge. But when checked at pixel 8 and pixel 9, these edges will be

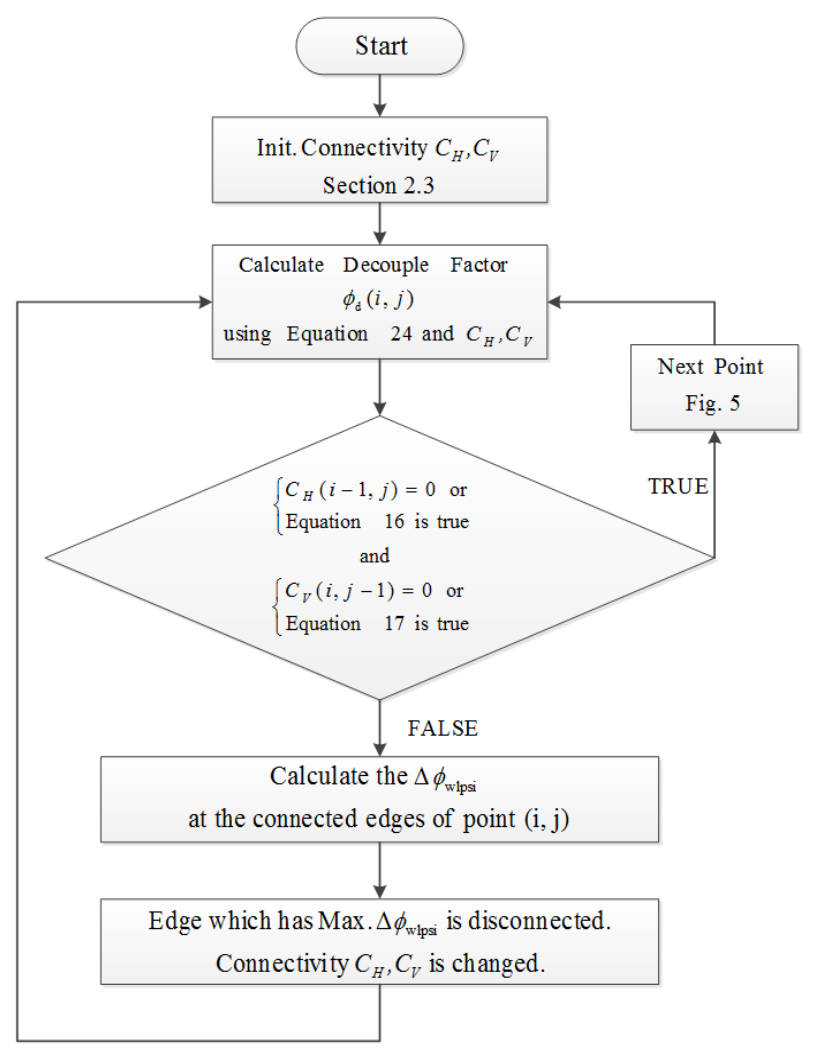

FIG. 4. Flow chart to check and modify the connectivity to satisfy Equation 16 and Equation 17. Upon the completion of this flow chart, we obtain one new decouple factor and the four new connectivity at point $(\mathrm{i}, \mathrm{j})$.

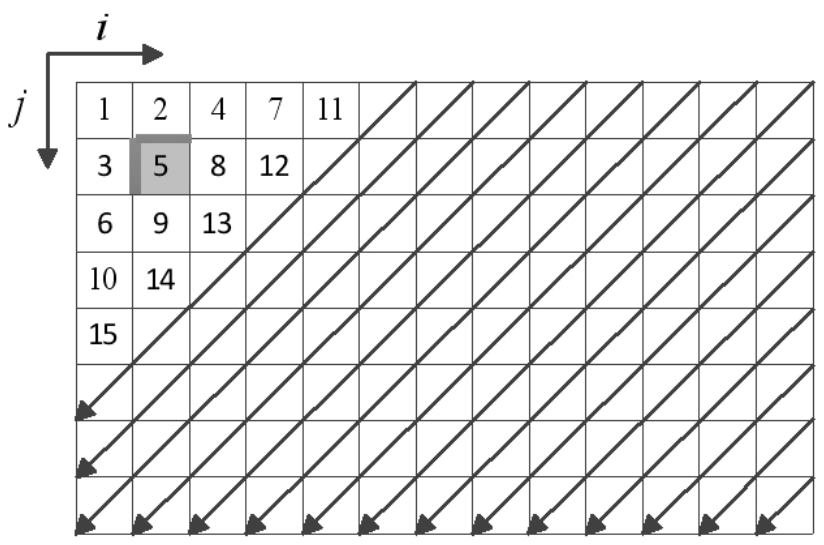

FIG. 5. The order of the flow chart in Figure 4 at two dimensional points. After completing the flow chart at all points, we obtain the new decouple factor map and the two new connectivity maps of the vertical map and the horizontal map. checked. This means that the decouple factor and the connectivity has been checked at all edges of pixel 5 . This calculation is repeated until $\phi_{d}(i, j)$ satisfies the condition equations, Eq. (16) and (17). This sequence is performed following the order shown in Fig. 5. The points which the edge connectivity changes from "connected" to "disconnected" demonstrate a major difference in $\tilde{\phi}_{w l p s i}$ between the neighboring points. Upon the completion this process, we obtain the decouple factor $\phi_{d}(i, j)$ at all points and the connectivity $C_{H}(i, j), C_{V}(i, j)$ at all edges.

\subsection{Phase Unwrap}

The remaining final step involves simply unwrapping the phase. In this paper, we use [10]a phase unwrapping method, which is modified to consider the decouple factor and the connectivity. A modified version of Herráez's method uses a phase of $\tilde{\phi}_{p s i, d}$, which is corrected with the decouple factor in place of the original phase $\phi_{p s i}$. The phase-unwrapping step is not performed at the disconnected edges identified by the connectivity. During the phase unwrapping procedure, stable phase unwrapping is possible because we use the decouple factor to compensate for the phase delay and the connectivity to suppress excessive phase unwrapping.

\section{EXPERIMENTS}

To examine the robustness to noise of our algorithm, a simulation was performed using the following procedure. The virtual step height with white noise in a range of $-k \pi$ to $k \pi$ was simulated as follows;

$$
\begin{aligned}
& \phi_{\text {wsi }}(i, j)=\left\{\begin{array}{lr}
0+\phi_{\text {noise }}(i, j) & 0 \leq i<50,0 \leq j<100 \\
\pi+\phi_{\text {noise }}(i, j) & 50 \leq i<100,0 \leq j<100
\end{array}\right. \\
& \phi_{\text {psi }}(i, j)=-\phi_{\text {noise }}(i, j) \\
& \phi_{\text {noise }}(i, j)=k \pi \cdot \operatorname{rand}(i, j)
\end{aligned}
$$

where $\operatorname{rand}(i, j)$ denotes a random signal with range of -1 to 1 and $k$ is the noise level. This noise can be considered for a simulated phase delay. The analysis proceeded with and without our fringe-order determination method. We discuss the fringe-order determination and if the fringeorder determination is incorrect at some pixel, the height error is exactly a multiple of the half of mean wavelength at that pixel. The virtual step height will be perfectly recovered if we obtain the correct fringe order. So the accuracy of noise simulation was not an important point in this noise simulation. We pointed out how many points fail to determinate the fringe order under noise. The results are shown in Fig. 6, showing the ratio of the points where unwrapping fails on the total points at a noise level ranging from $k=0$ to $k=3$. 
When the noise level $k$ is less than 1 , the results are identical between the WLPSI and our algorithm because there is no phase ambiguity. When the noise level range is from 1.0 to 2.0 , our algorithm gives a better result, as shown in Fig. 7. When the noise level, $k$, exceeds $2 \pi$, the

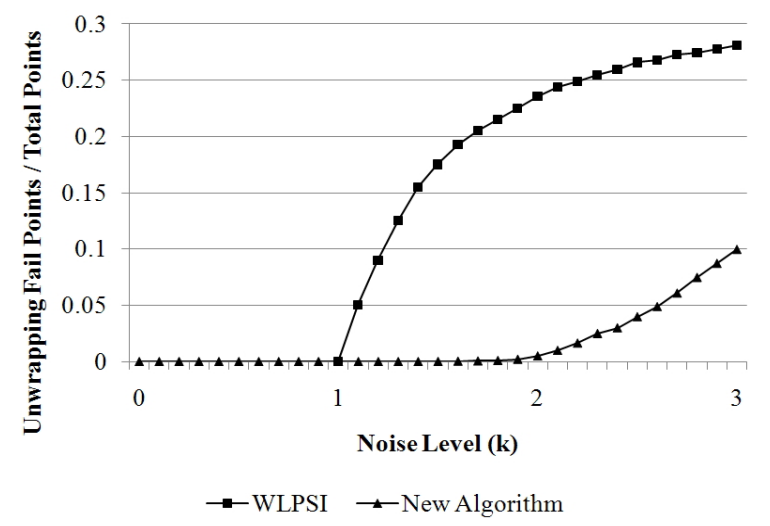

FIG. 6. We measured the simulation-surface induced random phase delay by Equation 25 with the conventional WLPSI and the new algorithm. The lower line shows the acceptable performance of the new algorithm.

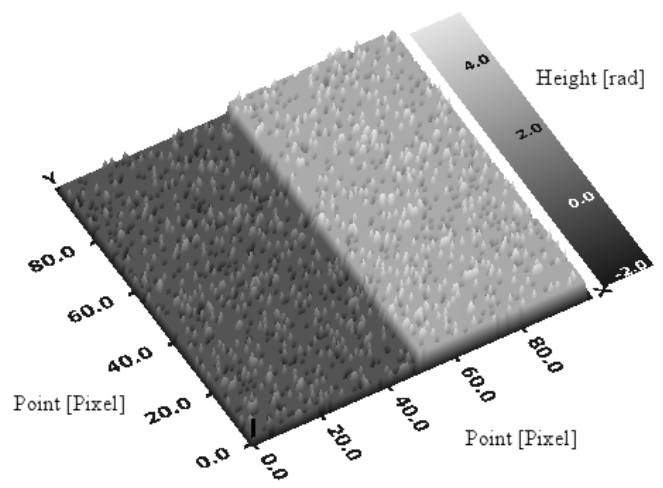

(a)

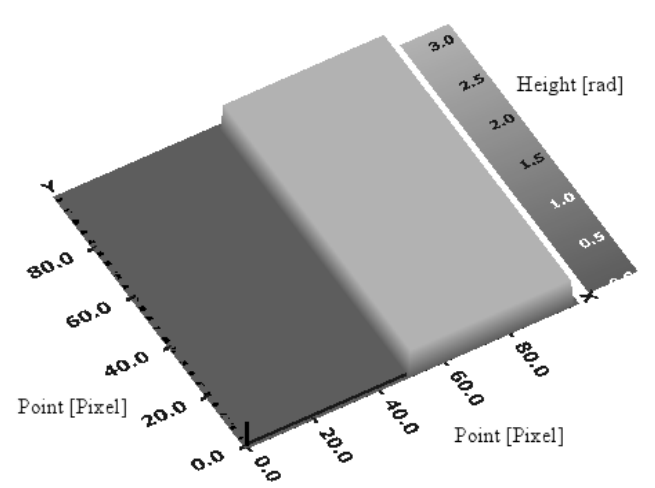

(b)

FIG. 7. The noise simulated surface measurement at $k=1.5$ : (a) WLPSI, (b) the proposed algorithm. Fig. 7(a) shows an instance of incorrect fringe-order determination. The noise's height is half of the mean wavelength. In Fig. 7(b), the new algorithm corrects the incorrect fringe-order determination completely. fringe order is not determined well enough under our algorithm due to the limited condition of the phase unwrapping in which the phase difference between successive points cannot exceed $2 \pi$ on a continuous surface. When the noise level is over 2 in an actual measurement, it can be estimated that the object was measured under too much vibration or at a steep angle.

When the measurement height is identical to or lower than the wavelength of the light source, the measurement result can be distorted by diffraction at the sharp edge of the step height, as noted in earlier research by, for instance, [5]. We measured the step height standard, VLSI SHS-4500, at a height of $470.3 \mathrm{~nm}$, which is quite close to the wavelength of light, with a 50X Mirau interference microscope. We intend to compare the result after we intentionally create the worst case with a large phase delay. In that case, the apparatus has a high-magnification lens and uses a Mirau-type lens. As shown in Fig. 8(a), we observed that the measured result was distorted at the edge of the step height standard without our fringe-order determination method. However as shown in Fig. 8(b), the measurement result was not distorted.

As shown in Fig. 9, another measurement test was performed with a Mirau interference microscope. We measured the LCD column spacer sample as shown in Fig. 9, which has a gradient on its surface with a phase delay of $\pi$. In particular, on the left side of the sample, the phase delay

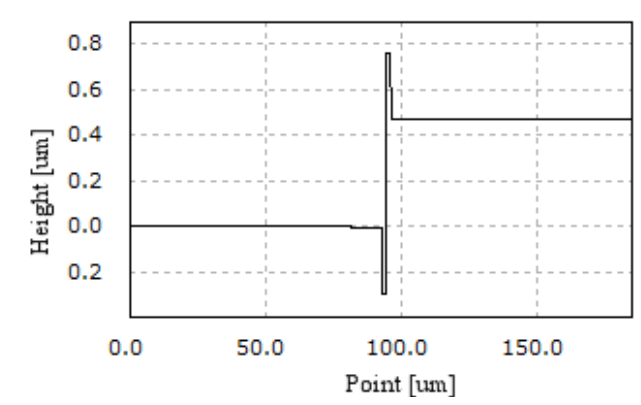

(a)

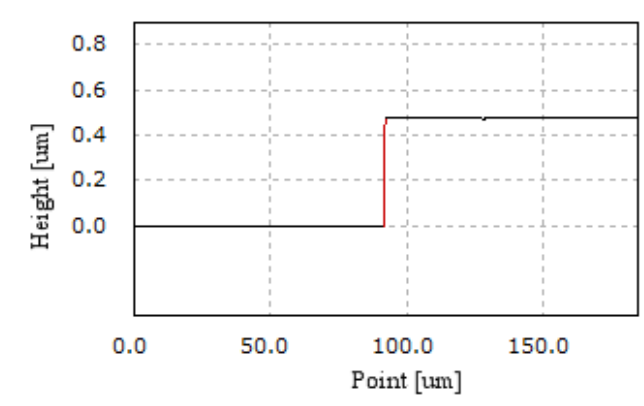

(b)

FIG. 8. Profiles of VLSI SHS-4500 at a height of $470.3 \mathrm{~nm}$ as measured with (a) WLPSI and (b) the proposed algorism using a 50X Mirau interference microscope. At a sharp edge of the step sample, the new algorithm can correct the incorrect fringe-order determination. 

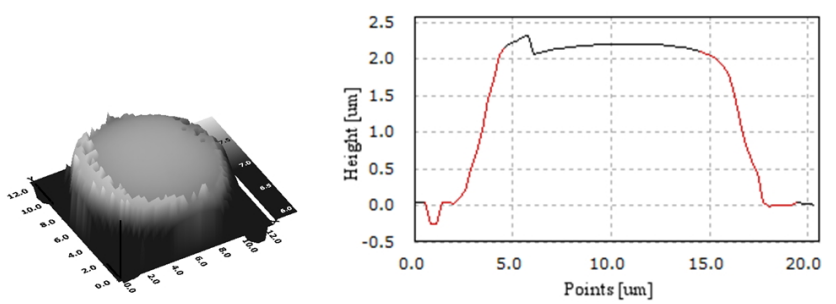

(a)
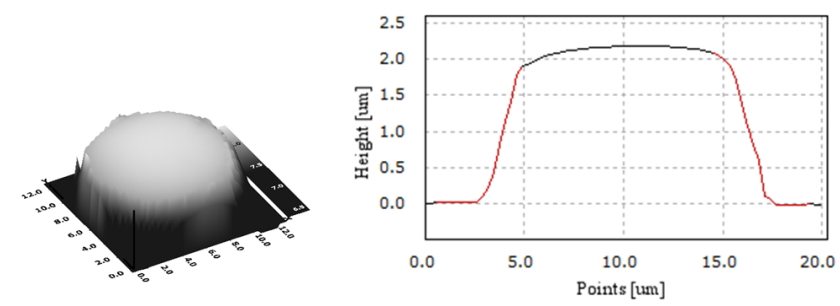

(b)

FIG. 9. 3D shapes and profiles of column spacer with a steep angle as measured by a) WLPSI and $b$ ) the proposed algorism using a 50X Mirau interference microscope. This result shows that the new algorithm corrects the incorrect fringe-order determination on the left side of the column spacer.

is greater than that on the right side due to the optic misalignment of the microscope. When applying phase unwrapping without our fringe-order determination method, the measurement result is distorted at the boundary of the sample, with a phase delay of more than $\pi$, as shown in Fig. 9(a). However, when applying with our fringe-order determination method, it was possible stably to unwrap a phase delay of more than $\pi$; in addition the measurement result is not distorted, leading to correct measurement result, as shown in Fig. 9(b).

\section{CONCLUSION \& DISCUSSION}

In this paper, a new algorithm is proposed for the fringe-order determination in the white light phase-shifting interferometry to compensate for the phase delay and to suppress excessive phase unwrapping to overcome the phase ambiguity problem.

(1) A decouple factor is proposed to compensate for the phase delay. When the phase is compensated for by the decouple factor prior to unwrapping, the WLPSI result can be successfully unwrapped, using the proposed Eq. (24) for calculating the decouple factor at all points.

(2) The concept of connectivity is proposed to suppress excessive phase unwrapping. The connectivity is defined in the vertical and the horizontal directions, and proper initial connectivity values are devised beforehand as explained in Section 2.3. This process allows the determination of the edges at which phase unwrapping is prohibited.

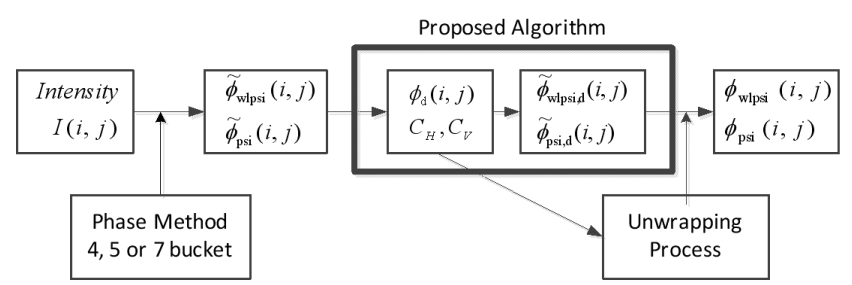

FIG. 10. Summarization of the proposed algorithm. The proposed algorithm does not modify the conventional WLPSI algorithm.

(3) The decouple factor and the connectivity are successfully calculated and corrected according to the proposed flow chart shown in Fig. 4.

(4) We performed a simulation under noise to verify the proposed method. The result showed that our method can reduce the errors induced by diffraction at a low step height close to the wavelength of light. We also measured a sample with a steep angle, with the result demonstrating that our method can successfully perform phase unwrapping for a phase delay of more than $\pi$.

(5) The proposed algorithm is found to be very robust against noise, diffraction, and the angle of the surface through measurement tests using white-light phaseshifting interferometry.

The proposed algorithm is summarized as shown in Fig. 10. This algorithm adds the decouple factor and the connectivity to the conventional WLPSI process which has the phaseunwrapping process. So our algorithm can be used in the conventional WLPSI which uses any phase method like the 4 bucket, 5 bucket and 7 bucket algorithm. The calculating time according to this algorithm is small. Some initial value calculation time and the flow chart calculation time of Fig. 4 are needed additionally. The flow chart calculation of Fig. 4 was processed one time for each pixel. So the number of total calculations is the same as the total number of pixels.

\section{REFERENCES}

1. G. S. Kino and S. S. C. Chim, "Mirau correlation microscope," Appl. Opt. 29, 3775-3783 (1990).

2. K. G. Larkin, "Efficient nonlinear algorithm for envelope detection in white light interferometry," J. Opt. Soc. Am. A 13, 832-843 (1996).

3. P. Sandoz, "An algorithm for profilometry by white-light phase-shifting interferometry," J. Mod. Opt. 43, 1545-1554 (1996).

4. P. Sandoz, R. Devillers, and A. Plata, "Unambiguous profilometry by fringe-order identification in white-light phaseshifting interferometry," J. Mod. Opt. 44, 519-534 (1997).

5. A. Harasaki and J. C. Wyant, "Fringe modulation skewing effect in white-light vertical scanning interferometry," Appl. Opt. 39, 2101-2106 (2000). 
6. A. Harasaki, J. Schmit, and J. C. Wyant, "Improved verticalscanning interferometry," Appl. Opt. 39, 2107-2115 (2000).

7. J. Schmit and A. Olszak, "High-precision shape measurement by white-light interferometry with real-time scanner error correction," Appl. Opt. 41, 5943-5950 (2002).

8. P. de Groot, X. C. de Lega, J. Kramer, and M. Turzhitsky, "Determination of fringe order in white-light interference microscopy," Appl. Opt. 41, 4571-4578 (2002).

9. K. Itoh, "Analysis of the phase unwrapping problem," Appl. Opt. 21, 2470-2470 (1982).
10. M. A. Herráez, D. R. Burton, M. J. Lalor, and M. A. Gdeisat, "Fast two-dimensional phase-unwrapping algorithm based on sorting by reliability following a noncontinuous path," Appl. Opt. 41, 7437-7444 (2002).

11. J.-H. Kim, S.-W. Yoon, J.-H. Lee, W.-J. Ahn, and H.-J. Pahk, "New algorithm of white-light phase-shifting interferometry pursing higher repeatability by using numerical phase error correction schemes of pre-processor, main processor, and post-processor," Optics and Lasers in Engineering 46, 140-148 (2008). 